\title{
Norois
}

Environnement, aménagement, société

\section{Les réorganisations hydrographiques dans le sud du Bassin parisien : l'exemple de la Bouzanne}

River diversion in the southern Paris basin: the Bouzanne example

Jean-Pierre Larue et Robert Étienne

\section{OpenEdition}

1 Journals

Édition électronique

URL : https://journals.openedition.org/norois/1384

DOI : 10.4000/norois. 1384

ISBN : 978-2-7535-1553-6

ISSN : $1760-8546$

Éditeur

Presses universitaires de Rennes

Édition imprimée

Date de publication : 1 décembre 2007

Pagination : 101-116

ISBN : 978-2-7535-0616-9

ISSN : 0029-182X

\section{Référence électronique}

Jean-Pierre Larue et Robert Étienne, «Les réorganisations hydrographiques dans le sud du Bassin parisien : l'exemple de la Bouzanne », Norois [En ligne], 205 | 2007/4, mis en ligne le 01 décembre 2009, consulté le 13 janvier 2022. URL : http://journals.openedition.org/norois/1384 ; DOI : https:// doi.org/10.4000/norois. 1384

(c) Tous droits réservés 


\title{
LES RÉORGANISATIONS HYDROGRAPHIQUES
}

\section{DANS LE SUd DU BASSIN PARISIEN : L'EXEMPLE DE LA BOUZANNE}

\author{
JEAN-Pierre LARUE \\ GÉodynamique des milieuX nATURELS ET DE L'ENVIRONNEMENT \\ (Université de Paris 12 - Val de Marne), \\ 61 avenue du Général-de Gaulle - 94010 CréTEIL cedex \\ larue@univ-paris12.fr \\ ROBERT ÉTIENNE \\ LABORATOIRE RHODANIEN DE GÉOMORPHOLOGIE \\ (Université Lumière - Lyon 2), \\ 5 avenue Pierre-Mendès-France -69676 Bron cedex
}

\section{RÉSUMÉ}

Cette étude vise à préciser les dates, les processus, les causes et les impacts géomorphologiques des changements de cours qui se sont produits entre la Creuse et l'Indre, dans le sud du Bassin parisien. L'analyse géomorphologique et sédimentologique permet de reconstituer les paléotracés successifs et de les replacer dans le cadre tectonique local, à savoir le soulèvement de la cuesta bajocienne et du dôme de Brion. La capture de la Bouzanne, qui est datée entre 200 et $280 \mathrm{ka}$ à partir des terrasses de la Creuse, est aussi due à l'érosion régressive induite par le creusement de la Creuse, plus rapide que celui de l'Indre. Les impacts géomorphologiques sont importants aussi bien dans la vallée conquérante que dans le système décapité. Ainsi, le recul des knickpoints développés suite à la capture s'est fait à des vitesses très variables en fonction de la résistance du substrat.

MotS CLÉ : Capture - Impacts géomorphologiques - Minéraux lourds - Néotectonique - Sud du Bassin parisien.

\section{ABSTRACT}

\section{River diversion in the southern Paris basin: the Bouzanne example}

This study attempts to assess the chronology, the processes and the geomorphological impacts of the river-bed diversions that have occurred between the Creuse and the Indre, in the southern part of the Bassin de Paris. Geomorphological and sedimentological analyses of alluvial deposits were carried out as the basis for correlating the different formations, and for reconstructing the palaeodrainage. The main causes of the diversions are the Pliocene and Pleistocene uplift of the Brion dome and of the "Accident sud du Bassin de Paris". The Bouzanne capture, which is dated between 200 and $280 \mathrm{ka}$ out of the Creuse terraces material, is also due to upward erosion driven by the Creuse incision which has been faster than that of the Indre. Significant impacts occur in the winner valley as well as in the beheaded system. The Bouzanne knickpoint retreat is controlled by lithology. 
KEY WORDS: Geomorphologic impacts - Heavy Minerals - Neotectonics - River Diversion - Southern Paris basin.

Depuis que W. M. Davis (1895) a introduit la notion de capture, l'intérêt des géomorphologues pour l'étude des réorganisations hydrographiques ne s'est guère démenti. La recherche des traces morphologiques de captures (coudes de capture, vallées mortes, ruptures de pente) a été complétée par l'analyse sédimentologique des dépôts conservés, permettant de reconstituer les profils longitudinaux des paléotracés successifs (Weisrock, 1997). Les études récentes insistent sur les impacts géomorphologiques à l'amont et à l'aval des captures (Mather, 2000a et b; Maher et al., 2007). Néanmoins, comme l'a souligné P. Bishop (1995), les processus précis des changements de cours demeurent souvent mal élucidés.

Dans le sud du Bassin de Paris, des changements de cours affectent toutes les rivières, de l'Auron à la Creuse (fig. 1), principalement là où elles traversent la cuesta de calcaire jurassique soulevée par l'« accident sud du Bassin de Paris » (Freytet, 1988; Freytet et al., 1986, 1989; Larue, 2000, 2005, 2008). Cette constatation confirme que les captures ne sont jamais isolées, mais toujours l'expression d'un remaniement général bien plus vaste d'origine structural (Weisrock, 1997). Elles se sont produites durant tout le Quaternaire : ainsi, la migration vers l'ouest de l'Arnon remonte au Pléistocène inférieur (2 Ma à 780 ka), alors que la capture de la Marmande par le Cher s'est réalisée juste avant le remblaiement weischsélien (Larue, 2000).

En utilisant la méthode morphométrique, P. Freytet (1988) et P. Freytet et al. (1986) ont montré que les déformations de la surface d'aplanissement antérieure à l'incision des cours d'eau étaient à l'origine d'importantes réorganisations hydrographiques dans le sud du Bassin parisien. Selon ces auteurs, la distension nord-sud qui a débuté vers $3 \mathrm{Ma}$ (Pliocène supérieur) serait à l'origine de la déviation vers l'ouest de toutes les rivières, de l'Auron à la Creuse. Ainsi, la Bouzanne a abandonné son cours septentrional (à la place de l'Indre actuelle) pour rejoindre la Creuse et le système Vauvre-Indre-Igneraie a emprunté la cluse d'Ardentes et déposé la formation d'Ardentes (fig. 2). La distension ouest-est qui fait suite a favorisé l'ouverture des fossés nord-sud et permis à l'ancienne Bouzanne septentrionale de capturer à la sortie de la cluse d'Ardentes le système Vauvre-Indre-Igneraie pour former l'Indre actuelle. L'infléchissement de la Creuse vers l'ouest à partir de Saint-Gaultier s'explique par un ensemble de failles de direction générale $\mathrm{N} 90^{\circ}$ dont les jeux successifs ont interrompu l'écoulement vers la Brenne, matérialisé par quelques placages alluviaux en forêt de Lancosme (Freytet et al., 1989). Cependant, les tracés exacts, la chronologie et les dates précises de ces réorganisations hydrographiques restent incertains. Aussi, les objectifs de ce travail sont-ils de préciser les dates, les processus et les causes des changements de cours entre la Creuse et l'Indre, et aussi d'analyser leurs impacts géomorphologiques.

\section{Cadre de l'étude}

Les rivières de la bordure nord du Massif central suivent des tracés méridiens jusqu'à la limite sud de la Brenne dans la partie occidentale et jusqu'au pied de la cuesta de calcaire jurassique dans le secteur oriental. L'Arnon, l'Indre et la Bouzanne naissent sur le versant nord des hauteurs d'Aigurande, aux altitudes respectives de 475 m, 480 m et 400 m. Le Cher et la Creuse prennent leurs sources plus au sud, à des altitudes plus élevées, respectivement dans le sud de la Combraille à $720 \mathrm{~m}$ d'altitude, et sur le plateau de Millevaches à $860 \mathrm{~m}$ (tableau 1). D'importantes bifurcations vers l'ouest se produisent dans le Boischaut et au sud de la Brenne, puis les cours se divisent en deux ensembles de part et d'autre du dôme de Brion : à l'est, ils convergent vers le nord et la Sologne, alors qu'à l'ouest, ils se dirigent vers le nord-ouest et la Touraine. 

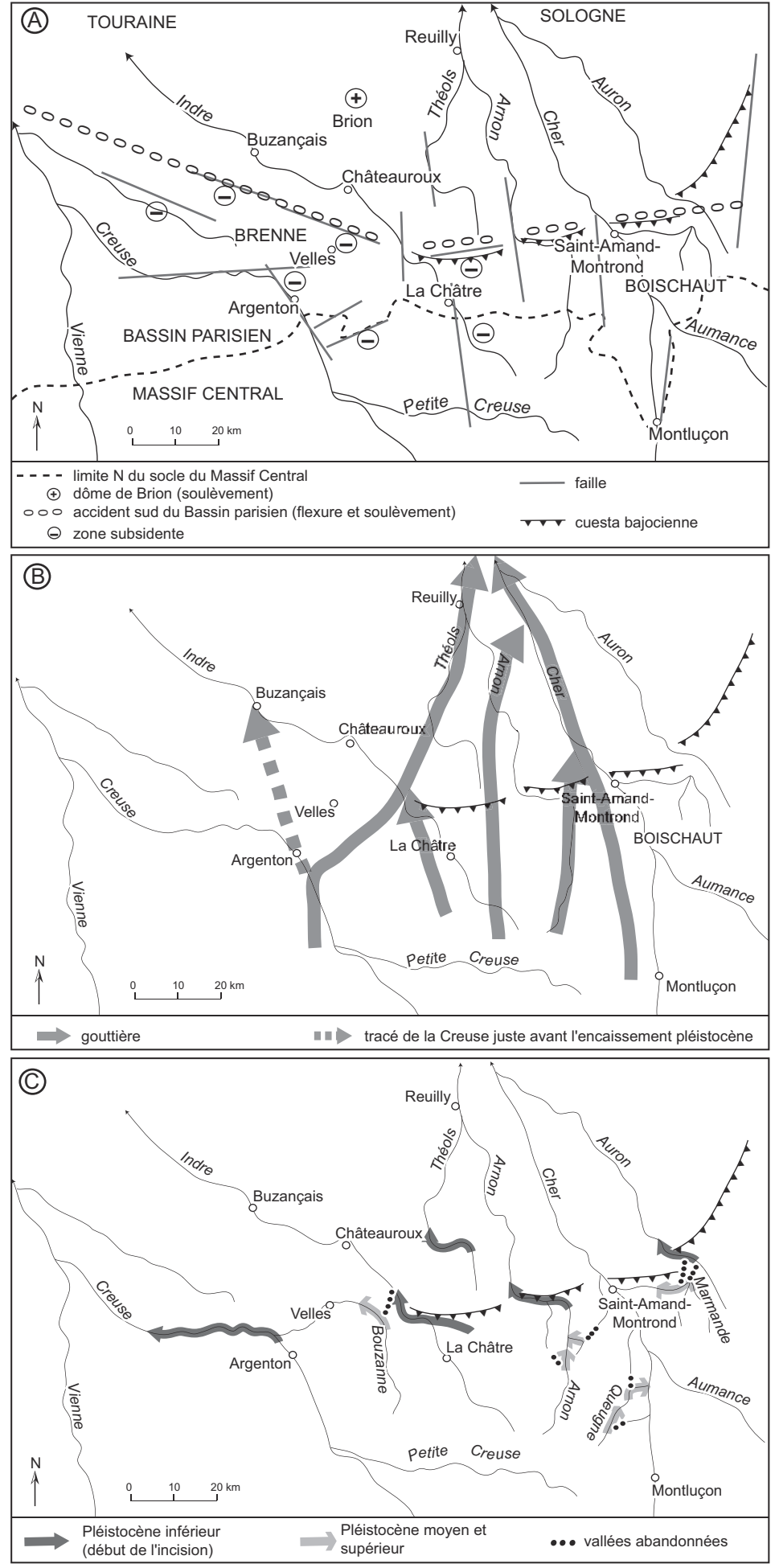

Figure 1 : Les principaux changements de cours dans le sud du Bassin parisien

A) Cadre structural. B) Paléotracés au Pliocène supérieur $(3,4$ à $2 \mathrm{Ma})$. C) Changements de cours pléistocènes $(2 \mathrm{Ma}$ à 10 ka).

The main hydrographic rearrangements in the southern Basin of Paris A) Structural framework. B) Upper Pliocene palaeocourses $(3,4$ to $2 \mathrm{Ma})$. C) Pleistocene captures (2 Ma to $10 \mathrm{ka})$. 


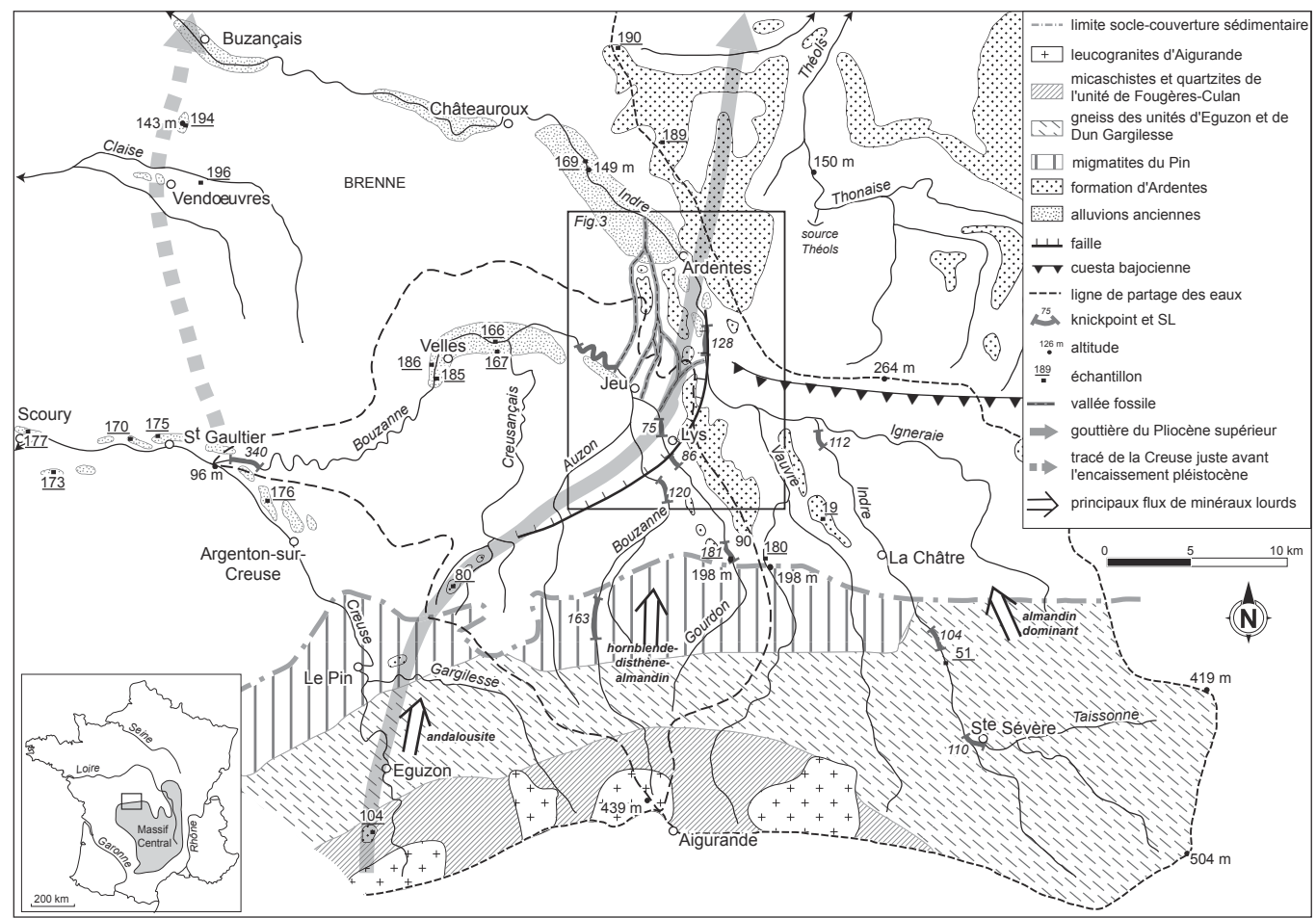

Figure 2 : Carte de localisation des formations alluviales et des échantillons prélevés dans la région d'Ardentes Location map of alluvial deposits and samples in the Ardentes district

\begin{tabular}{|c|c|c|c|c|}
\hline cours d'eau & $\begin{array}{c}\text { altitude des } \\
\text { sources }(\mathrm{m})\end{array}$ & longueur $(\mathrm{km})$ & $\begin{array}{c}\text { pente } \\
\text { moyenne }(\mathrm{m} / \mathrm{km})\end{array}$ & $\begin{array}{c}\text { indice de } \\
\text { concavité }\end{array}$ \\
\hline Arnon & 475 & 118 & 3,07 & 0,66 \\
Bouzanne amont & 400 & 28,7 & 8,57 & 0,48 \\
Bouzanne & 400 & 60 & 5,16 & 0,61 \\
Cher & 720 & 293 & 2,92 & 0,76 \\
Creusançais & 230 & 23 & 4,35 & 0,35 \\
Creuse & 860 & 222 & 3,53 & 0,79 \\
Gourdon & 325 & 22,9 & 7,43 & 0,59 \\
Indre amont & 480 & 58,5 & 5,67 & 0,72 \\
Indre & 480 & 209 & 2,15 & 0,75 \\
\hline
\end{tabular}

Tableau 1 : Caractéristiques des cours d'eau

\section{Rivers characteristics}

Ces cours d'eau ont des régimes pluvio-évaporaux océaniques avec de hautes eaux l'hiver et de basses eaux l'été. À Ardentes (697 km² de bassin), le module moyen de l’Indre est de $5,39 \mathrm{~m}^{3} / \mathrm{s}$, les hautes eaux de février atteignent $11,40 \mathrm{~m}^{3} / \mathrm{s}$ et les basses eaux d'août $1,23 \mathrm{~m}^{3} / \mathrm{s}$, les crues quinquennales peuvent dépasser $94 \mathrm{~m}^{3} / \mathrm{s}$ et les étiages quinquennaux descendre en dessous de $0,32 \mathrm{~m}^{3} / \mathrm{s}$ (ces données hydrologiques couvrant la période 1969-2006 proviennent de la Banque Hydro, DIREN Centre). À Velles (434 $\mathrm{km}^{2}$ de bassin), le module de la Bouzanne est de 3,19 m³/s, les hautes eaux de février atteignent $7,18 \mathrm{~m}^{3} / \mathrm{s}$ et les basses eaux d'août $0,61 \mathrm{~m}^{3} / \mathrm{s}$, les crues quinquennales peuvent 
dépasser $67 \mathrm{~m}^{3} / \mathrm{s}$ et les étiages quinquennaux descendre en dessous de $0,21 \mathrm{~m}^{3} / \mathrm{s}$. Les modules spécifiques des deux cours d'eau sont presque identiques : 7,7 l/s/ $/ \mathrm{km}^{2}$ pour l'Indre qui écoule une lame d'eau de $245 \mathrm{~mm}$ par an et 7,4 l/s/ $\mathrm{km}^{2}$ pour la Bouzanne qui évacue $233 \mathrm{~mm}$ par an. La Creuse, qui a capturé la haute Bouzanne, est un cours d'eau plus puissant : à Scoury (3343 km² de bassin), elle enregistre un module moyen de $36,4 \mathrm{~m}^{3} / \mathrm{s}$ et un module spécifique de $10,9 \mathrm{l} / \mathrm{s} / \mathrm{km}^{2}$, soit un écoulement de $344 \mathrm{~mm}$ par an.

Au sud, le domaine du plateau d'Aigurande correspond à une série cristallophyllienne ployée au Dévonien en une vaste antiforme est-ouest dont l'axe est jalonné de batholites de leucogranites à deux micas. Quatre unités métamorphiques sont distinguées : celle de Fougères-Culan est constituée de micaschistes et de quartzites, celles d'Éguzon et de Dun-Gargilesse sont gneissiques et celle du Pin est migmatitique (Cohen-Julien et al., 1998). Au nord, le socle plonge lentement sous la couverture sédimentaire secondaire qui présente une structure monoclinale avec un pendage moyen de $1 \%$ vers le nord, accentué localement par quelques failles et flexures, comme au niveau de l'« accident sud du Bassin de Paris » qui s'étire d'Ancenis à Saint-Amand-Montrond (Lorenz et Lorenz, 1985) (fig. 1 et 2). À l'ouest d'Ardentes, cet accident suit une direction sud armoricaine $\left(\mathrm{N} 110^{\circ}\right)$ et rejoue en limite de blocs basculés depuis le Jurassique, ce qui provoque à chaque reprise une surrection du bloc septentrional (Freytet et al., 1989). Le soulèvement du dôme de Brion et la subsidence du demi-fossé d'effondrement de Velles résultent de ces mouvements tectoniques. Ces déformations et la lithologie variée, conglomérats, grès, argiles et marnes triasiques, marnes, argiles et calcaires du Jurassique inférieur et calcaires du Jurassique moyen, ont favorisé la formation de la cuesta bajocienne, bien développée au nord de Saint-Amand-Montrond. La dépression subséquente du Boischaut, entre Argenton-sur-Creuse et Saint-Amand-Montrond est dégagée dans les marnes liasiques. Des sédiments tertiaires sont conservés localement sur le socle et sur la couverture secondaire. Étalés sur une surface rendue irrégulière par l'érosion karstique des calcaires jurassiques, les dépôts de Brenne ont été datés par J.-J. Châteauneuf (1977) entre l'Yprésien et le Ludien. À la base de la formation, les galets de quartz de $10 \mathrm{~cm}$ de diamètre et les chailles à patine noire d'origine organique témoignent de transports à forte compétence. Les formations mio-pliocènes recouvrent parfois ces dépôts, comme dans le bassin d'angle de faille de Lys-Saint-Georges, situé plus à l'est, au sud d'Ardentes. Ce bassin de type «pull-apart » a conservé trois générations de dépôts superposés (Lorenz et al., 1985). Les premiers dépôts, issus du démantèlement des assises secondaires environnantes, sont formés de grés, d'argiles blanches à quartz et chailles à patine noire, puis d'argiles et marnes et sont antérieurs au calcaire blanc lacustre éocène supérieur qui les recouvre. Au-dessus, les argiles rouges à petits quartz de la formation des buttes de Jeu et les sables fins micacés de Tranzault témoignent d'apports lointains du socle, probablement au Miocène. Enfin, ravinant les précédents, la formation d'Ardentes à sables, graviers et galets de quartz et chailles résulte d'apports chenalisés au Pliocène supérieur (Lablanche et al., 1994), comme on en rencontre dans le bassin du Cher (Larue et Étienne, 1998).

En Brenne, des placages d'alluvions ne dépassant pas $2 \mathrm{~m}$ d'épaisseur se trouvent en position culminante vers $126 \mathrm{~m}$ à l'ouest de Vandœuvres et à $143 \mathrm{~m}$ entre Vandœuvres et Buzançais (Rasplus et al., 1989a). Il s'agit de graviers et galets de quartz et de roches siliceuses emballés dans une matrice argilo-sableuse qui seraient issus de l'altération et du remaniement par ruissellement d'alluvions anciennes pouvant se raccorder aux unités Ft ou Fu (Pléistocène inférieur) conservées dans la vallée de la Creuse entre Saint-Gaultier et Le Blanc (Rasplus et al., 1989b).

\section{Méthodes}

L'étude géomorphologique et sédimentologique des dépôts alluviaux a permis de raccorder les différentes nappes alluviales de l'amont à l'aval et ainsi de reconstituer les paléotracés successifs. Des échantillons ont été prélevés dans le lit mineur des cours actuels et dans les alluvions anciennes conservées en terrasses (fig. 2 et 3), à la faveur de coupes naturelles ou de forages à la tarière permettant d'atteindre le matériau en place. Concentrés selon la méthode densimétrique 


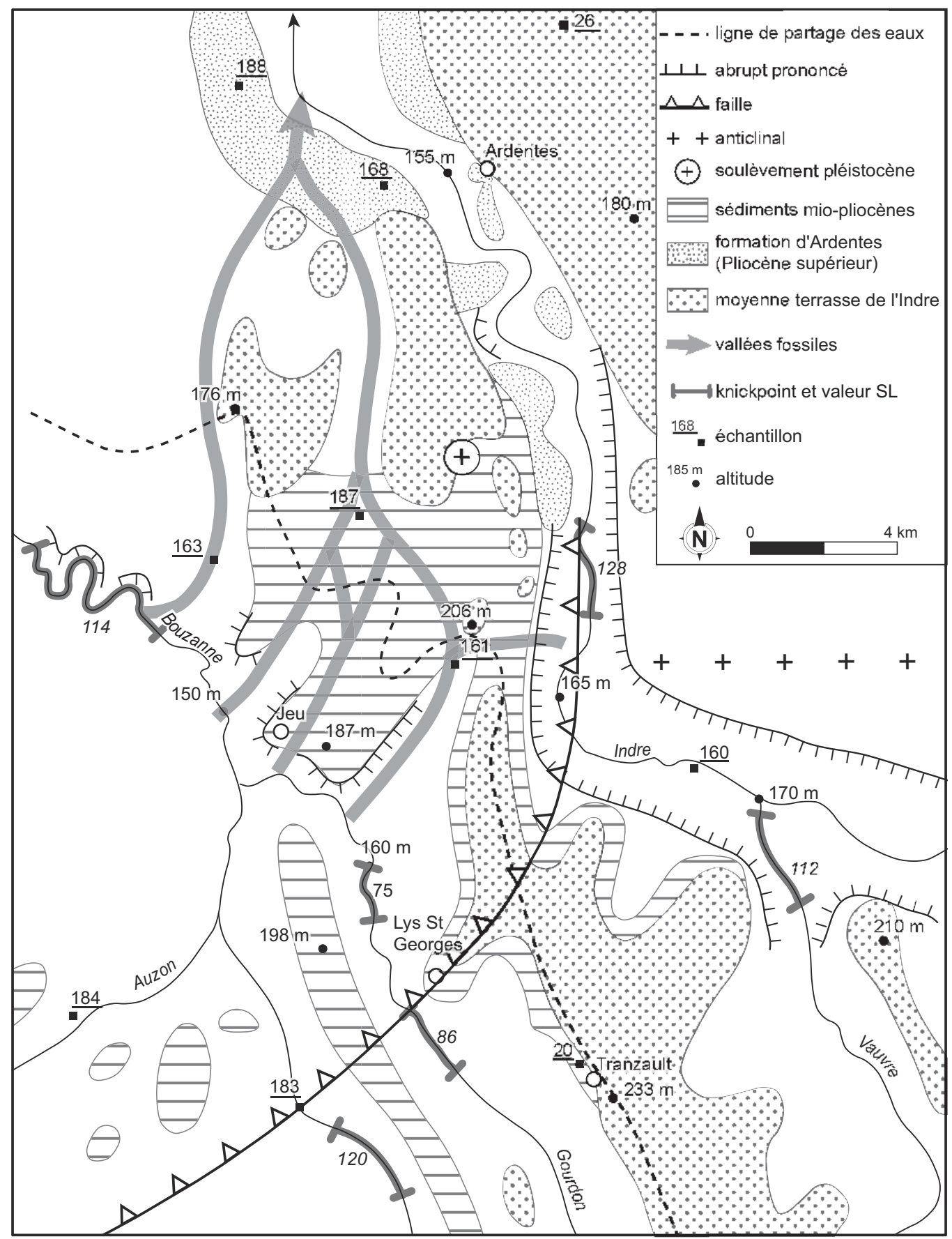

Figure 3 : Agrandissement du secteur de capture de la Bouzanne Enlargement of the Bouzanne capture area 
de séparation au bromoforme (Parfenoff et al., 1970), les minéraux lourds ont été déterminés au microscope optique (tableau 2). Les pourcentages de minéraux transparents ont été calculés à partir de l'analyse d'environ 200 grains.

\begin{tabular}{|c|c|c|c|c|c|c|}
\hline $\mathrm{n}^{\circ}$ éch & lieu-dit & carte $1 / 50000$ & coordonnées & $\begin{array}{l}\text { altitude } \\
(\mathrm{m})\end{array}$ & $\begin{array}{l}\text { profondeur } \\
(\mathrm{m})\end{array}$ & lithostratigraphie \\
\hline 19 & W La Châtre & La Châtre & $572-177,1$ & 255 & 1 & sables argileux \\
\hline 20 & Tranzault & Ardentes & $562,5-181,2$ & 225 & 1,50 & sables micacés \\
\hline 26 & Sanguille & Ardentes & $561,3-197,5$ & 167 & 1,50 & alluvions (FA) \\
\hline 32 & W Reuilly & Vatan & $576,5-231,2$ & 158 & 0,80 & alluvions ocre-rouille \\
\hline 33 & SW Reuilly & Vatan & $576,7-230,4$ & 154 & 0,60 & sables argileux rouges \\
\hline 51 & Verneau & La Châtre & $577,7-168,8$ & 236 & & alluvions actuelles de l'Indre \\
\hline 80 & butte Gravelle & Argenton-sur-Creuse & $545,6-173,7$ & 235 & 2 & sables argileux $(\mathrm{Fp})$ \\
\hline 104 & N La Feyte & Dun-le-Palestel & $543,3-158,2$ & 289 & 0,80 & argile sableuse ocre \\
\hline 160 & Mers-sur-Indre & Ardentes & $564,7-185,4$ & 170 & & alluvions actuelles de l'Indre \\
\hline 161 & vallée fossile & Ardentes & $561,3-186,9$ & 176 & 0,60 & alluvions \\
\hline 163 & La Feuge & Velles & $557,8-188,2$ & 166 & 0,60 & alluvions \\
\hline 166 & E Arthon & Velles & $552,7-189,6$ & 136 & & alluvions actuelles de la Bouzanne \\
\hline 167 & E Arthon & Velles & $552,7-189,5$ & 145 & 1 & alluvions $(\mathrm{Fx})$ \\
\hline 168 & Ardentes & Ardentes & $560,7-193,4$ & 164 & 1 & alluvions $(\mathrm{Fx})$ \\
\hline 169 & SE Châteauroux & Velles & $555,4-198,8$ & 149 & & alluvions actuelles de l'Indre \\
\hline 170 & NE Rivarennes & Saint-Gaultier & $527,5-182,6$ & 95 & 2 & alluvions $(\mathrm{Fx})$ \\
\hline 173 & Pezay-le-Joli & Saint-Gaultier & $522,9-181$ & 155 & 0,60 & alluvions $(\mathrm{Ft})$ \\
\hline 175 & N Saint-Gaultier & Saint-Gaultier & $528,7-182,8$ & 126 & 2 & alluvions $\left(\mathrm{Fv}_{\mathrm{v}}\right)$ \\
\hline 176 & Saint-Marcel, & Argenton-sur-Creuse & $536,1-179,1$ & 130 & 1 & alluvions $\mathrm{F}_{\mathrm{W}}$ \\
\hline 177 & Scoury & Saint-Gaultier & $519,5-182,3$ & 105 & 1 & alluvions ( $\left.\mathrm{F}_{\mathrm{w}}\right)$ \\
\hline 180 & Le Ponderon & La Châtre & $566,5-175,2$ & 198 & & alluvions actuelles de la Vauvre \\
\hline 181 & N Fougerolles & La Châtre & $564,6-175,3$ & 198 & & alluvions actuelles du Gourdon \\
\hline 183 & $\begin{array}{l}\text { S Le Lys-Saint- } \\
\text { Georges }\end{array}$ & Ardentes & $559,8-180,4$ & 171 & & alluvions actuelles de la Bouzanne \\
\hline 184 & SE Buxières d'Aillac & Velles & $555,7-182$ & 165 & & alluvions actuelles de l'Auzon \\
\hline 185 & Forges & Velles & $545,7-185,3$ & 125 & & alluvions actuelles de la Bouzanne \\
\hline 186 & N Forges & Velles & $546-186,2$ & 132 & 0,60 & alluvions $(\mathrm{Fx})$ \\
\hline 187 & Paris & Ardentes & $559,5-188,4$ & 173 & 0,60 & alluvions $(\mathrm{Fx})$ \\
\hline 188 & SW La Clavière & Velles & $558-195,4$ & 160 & 0,60 & alluvions $\left(\mathrm{F}_{\mathrm{w}}\right)$ \\
\hline 189 & Bois des Fineaux & Châteauroux & $558,3-206,1$ & 160 & 0,60 & alluvions (FA) \\
\hline 190 & NE Montierchaume & Châteauroux & $557,2-209$ & 162 & 0,60 & alluvions (FA) \\
\hline 194 & L'Égaillé & Buzançais & $523,4-205,5$ & 143 & 0,60 & alluvions $(\mathrm{Ft})$ \\
\hline 196 & SE Claise & Velles & $532,4-199,8$ & 131 & 1,30 & alluvions $\left(\mathrm{Fw}_{w}\right)$ de la Claise \\
\hline
\end{tabular}

Tableau 2 : Localisation et lithostratigraphie des échantillons prélevés Location and lithostratigraphy of samples

Pour déterminer les causes tectoniques, les changements de cours ont été confrontés avec le canevas tectonique de la région établi par G. Lerouge (1984), G. Lerouge et al. (1986), C. Lorenz et J. Lorenz (1983 et 1985) et P. Freytet et al. (1985, 1986 et 1989). La datation des terrasses de la vallée de la Creuse (Despriée et al., 2004) a permis de préciser le moment de la capture de la Bouzanne. Les impacts morphologiques de cette capture ont été analysés dans les trois secteurs perturbés, c'est-à-dire, i) dans les vallées à l'amont de la capture, ii) dans la vallée conquérante de la Bouzanne à l'aval de la capture, et iii) dans la vallée de l'Indre qui perdu les apports de la haute Bouzanne. Pour cela, les profils longitudinaux des cours d'eau ont été construits à partir des cartes topographiques IGN (Institut Géographique National) au 1/25000, en utilisant les points cotés 
et l'altitude de chaque isohypse recoupant le talweg. L'équidistance des courbes de $5 \mathrm{~m}$ permet d'obtenir des profils suffisamment précis pour déceler les variations de pente et les principales ruptures de pente qui correspondent aux secteurs à pente anormalement forte, c'est-à-dire là où la pente augmente en créant une convexité locale dans la concavité générale du profil longitudinal. Le graphique adimensionnel H/Ho (ratio des altitudes) et L/Lo (ratio des longueurs) (fig. 4) permet de superposer et ainsi de comparer les profils de drains de longueur différente (Demoulin, 1998) ; H est l'altitude du cours d'eau au point mesuré, Ho est la dénivellation entre la source et l'exutoire, L est la distance à l'exutoire du point mesuré et Lo est la longueur du cours d'eau. Pour évaluer et comparer les concavités des profils, nous avons utilisé l'indice de concavité de W.B. Langbein (1964) : IC = 2A/H avec $\mathrm{A}=$ différence d'altitude entre le profil à mi-parcours et une ligne droite joignant les deux extrémités du profil, $\mathrm{H}=$ dénivellation entre la source et l'exutoire du drain. Le profil tend à être rectiligne quand la valeur de IC est proche de 0 ; à l'inverse, la concavité devient très forte quand la valeur approche de 1. Les ruptures de pente ont été systématiquement repérées en notant la dénivelée, la pente, la distance des sources, la lithologie, la présence de failles, de confluences et de méandres. Les corrélations entre ces éléments ont été établies. Le calcul des indices SL (longueur-pente) de E. A. Keller et N. Pinter (1996) : $\mathrm{SL}=(\Delta \mathrm{H} / \Delta \mathrm{L})$. L où $\Delta \mathrm{H} / \Delta \mathrm{L}$ est la pente du segment considéré et $\mathrm{L}$ la longueur du chenal en amont du point à partir duquel l'indice est calculé, permet de souligner les moindres ruptures de pente et les très fortes ou très faibles valeurs témoignent de déformations tectoniques si elles ne sont pas corrélables avec des facteurs lithologiques. La forte amplitude des valeurs permet de caractériser tous les changements de pente le long des profils longitudinaux; néanmoins, il faut savoir que pour des secteurs de pente identique, les valeurs augmentent avec la distance des sources.

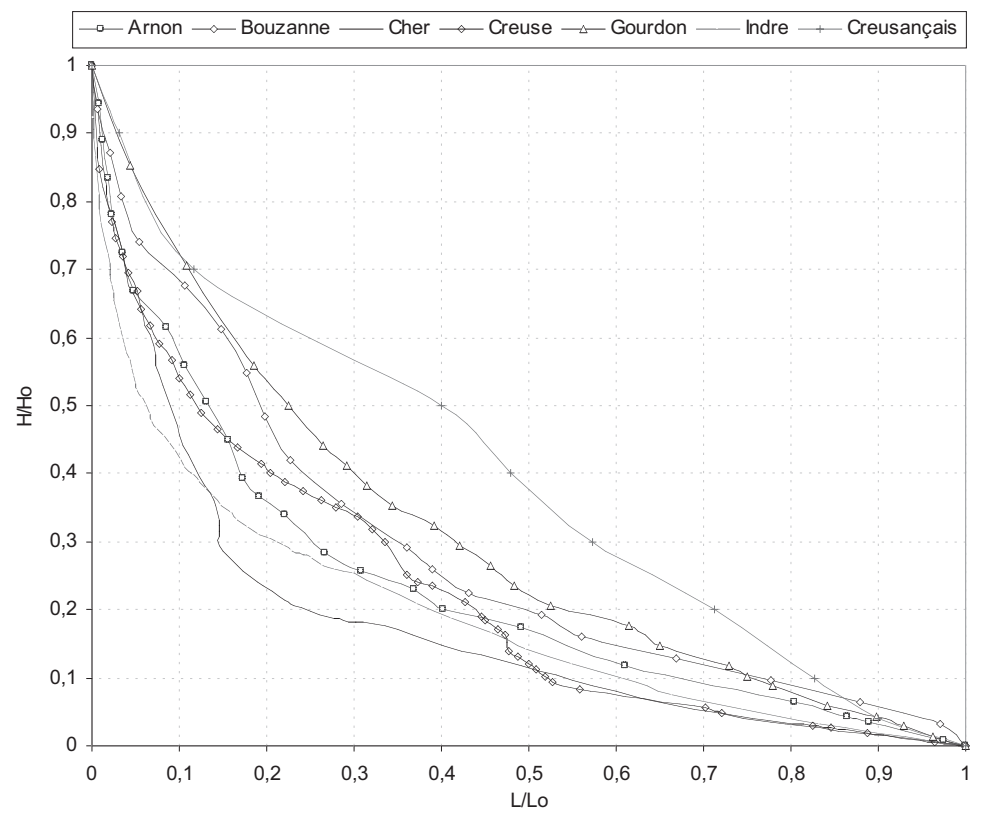

Figure 4 : Profils longitudinaux comparés H/Ho (ratio des altitudes) et L/Lo (ratio des longueurs) distance)

Dimensionless curves $\mathrm{H} / \mathrm{Ho}$ and L/Lo (ratio of altitude and ratio of 


\section{Résultats}

Certains minéraux lourds marqueurs permettent de reconstituer les principaux paléotracés (tableau 3, figs. 2 et 5). Ainsi, l'andalousite jalonne une gouttière que l'on peut suivre de la vallée de la Creuse (échantillon 104, au sud d'Éguzon), à celle de l'Arnon (échantillons 32 et 33, à Reuilly), en passant par la butte de Gravelle (échantillon 80), le bassin de Lys-Saint-Georges et la région d'Ardentes (échantillon 26).

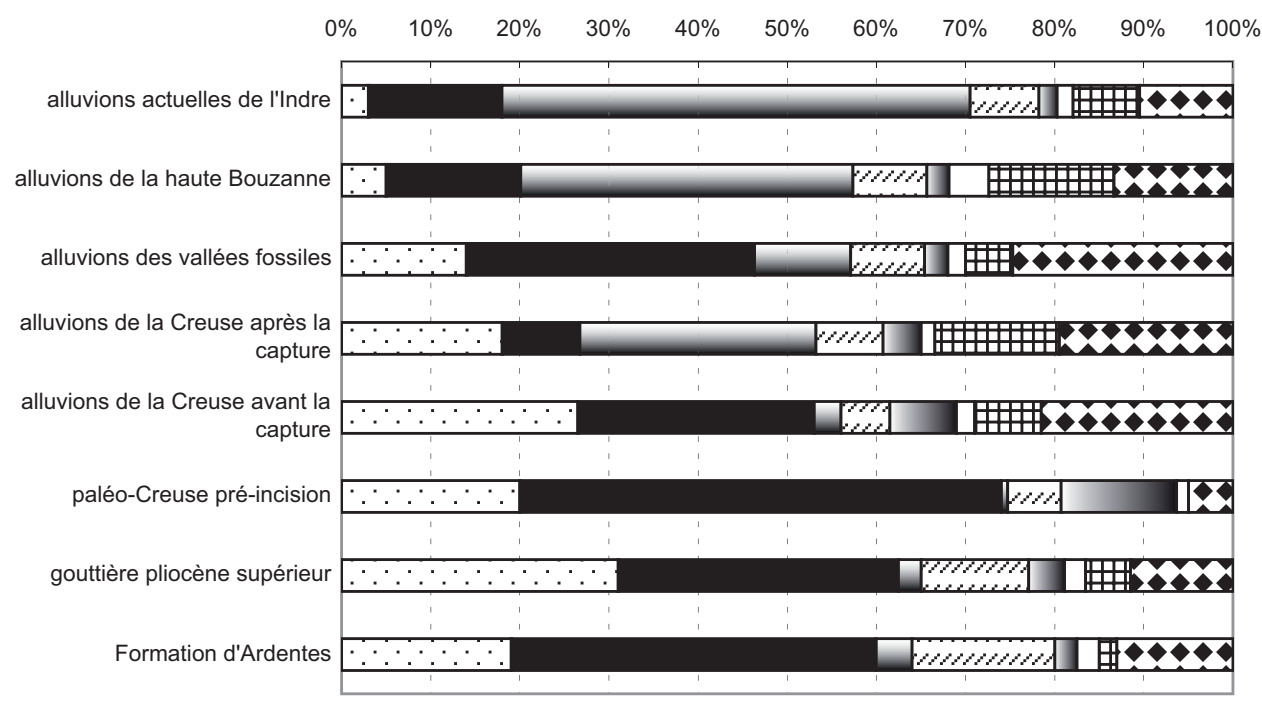

$\square$ zircon $\square$ tourmaline $\square$ almandin $[$ andalousite $\square$ staurotide $\square$ disthène $\boxplus$ hornblende $\square$ autres

Figure 5 : Composition en minéraux lourds des différentes formations alluviales Composition in heavy minerals of each alluvial formation

Les apports de la haute Bouzanne et de la haute Indre se distinguent par une composition en minéraux lourds sensiblement différente. Les cours d'eau principaux du bassin amont de la Bouzanne (Auzon, Gourdon et Bouzanne) sont caractérisés par la relative abondance de la hornblende et du disthène et par une teneur en almandin inférieure à $40 \%$ (échantillons 181, 183 et 184), alors que ceux du bassin amont de l'Indre (Taissonne, Vauvre et Indre) sont plus riches en almandin (toujours plus de $60 \%$ dans les échantillons 51 et 160). Les alluvions Fw (Saalien) de la vallée de l'Indre, entre Ardentes et Châteauroux (échantillon 188), ont une composition assez semblable à celle de la haute Bouzanne : elles se sont donc accumulées avant la réalisation de la capture. Dans la vallée de la Creuse, l'étagement des terrasses permet de préciser la date de cette dernière. Avant la capture, les alluvions de la Creuse ne renferment que très peu d'almandin (par exemple, 4 \% dans le niveau Fv (Elstérien ?) de Saint-Gaultier, échantillon 175), après cette dernière la teneur en almandin augmente brutalement (30 \% dans la moyenne terrasse $\mathrm{Fw}_{\mathrm{w}}$ de Scoury, échantillon 177). La capture s'est donc produite au début de l'accumulation de la nappe Fw qui a été datée entre 200 et 280 ka par la méthode de résonance paramagnétique électronique (Despriée et al., 2004).

Les profils longitudinaux de l'Indre, de la Bouzanne, du Gourdon et de l'interfluve BouzanneIndre (fig. 6) indiquent que, près du coude de la capture, le réseau de la Bouzanne est situé 16 m en contrebas de celui de l'Indre et que le lit de la Bouzanne anté-capture est maintenant perché 


\begin{tabular}{|c|c|c|c|c|c|c|c|c|c|c|c|c|c|c|c|c|c|c|c|c|}
\hline :气 & 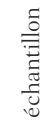 & ¿্ర & 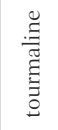 & $\stackrel{0}{\Xi}$ & 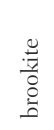 & 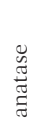 & $\frac{\tilde{0}}{0}$ & 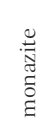 & 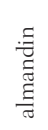 & 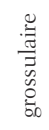 & $\stackrel{\mathscr{\Xi}}{\stackrel{\Xi}{\Xi}}$ & 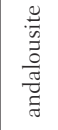 & 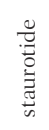 & $\begin{array}{l}\stackrel{\mathscr{\Xi}}{\tilde{\Xi}} \\
\stackrel{\Xi}{\Xi} \\
\overline{\bar{n}}\end{array}$ & $\frac{\mathscr{E}}{\stackrel{\Xi}{ \pm}}$ & $\begin{array}{l}\frac{0}{0} \\
\frac{0}{0} \\
\frac{0}{2}\end{array}$ & 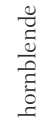 & 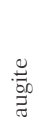 & $\begin{array}{l}\tilde{N} \\
\tilde{\Xi}\end{array}$ & $\frac{\stackrel{\Xi}{0}}{\frac{c}{c}}$ \\
\hline \multirow{5}{*}{$\begin{array}{l}\text { formation } \\
\text { d'Ardentes }\end{array}$} & 19 & 51 & 27 & 9 & 7 & & & & & 3 & & 3 & & & 5 & & l & & & \\
\hline & 20 & 3 & 64,5 & & 3 & & & 1 & 1 & 1 & & 21,5 & 2 & & 2 & & & & & \\
\hline & 26 & 11 & 48,5 & 3,5 & 2,5 & & 1 & & 1 & 2,5 & & 26,5 & 1 & & 1,5 & 1 & & & & \\
\hline & 189 & 7,5 & 36 & 4 & & & & 2 & 6 & & 7,5 & 17 & 4 & 4 & 2 & 7,5 & 2 & & 2 & \\
\hline & 190 & 22 & 29 & 2 & & & & & 8 & 2 & & 12 & 6 & & 2 & 8 & 4 & & 4 & \\
\hline \multirow{5}{*}{$\begin{array}{l}\text { gouttière } \\
\text { pliocène } \\
\text { supérieur }\end{array}$} & 32 & 29 & 28 & 3 & 10,4 & & 1 & 2 & 2 & 1 & & 10 & 9 & & 2 & 1 & & & & \\
\hline & 33 & 67 & 6 & 4 & 2 & & & 5 & & 2 & & 6 & 6 & & & 1 & & & & \\
\hline & 80 & 33 & 43 & 1 & & 1 & & 1 & 1,5 & & & 7 & 2 & & & 2 & 8 & & & \\
\hline & 104 & 37 & 28 & 1 & & 1 & & & 3 & & 7 & 6 & & & 6 & & 15 & & & \\
\hline & 189 & 7,5 & 36 & 4 & & & & 2 & 6 & & 7,5 & 17 & 4 & 4 & 2 & 7,5 & 2 & & 2 & \\
\hline $\begin{array}{l}\text { Creuse pré- } \\
\text { incision }\end{array}$ & 194 & 20 & 54 & 3,3 & & & & & 0,7 & & 2 & 6 & 13 & & 1,3 & & & & & \\
\hline \multirow{2}{*}{$\begin{array}{c}\text { alluvions de } \\
\text { la Creuse } \\
\text { avant } \\
\text { capture }\end{array}$} & 173 & 29 & 27 & 3 & & & & 2 & 2 & & 3 & 5 & 13 & & 3,5 & 3 & 6 & & & 0,5 \\
\hline & 175 & 24 & 26 & & & 1 & & & 4 & & 11 & 6 & 2 & & & 9 & 9 & & 7 & 2 \\
\hline \multirow{2}{*}{$\begin{array}{c}\text { alluvions de } \\
\text { la Creuse } \\
\text { après } \\
\text { capture }\end{array}$} & 170 & 20 & 13 & & & & & & 23 & & 17 & 6 & 2,5 & & 1 & 1 & 13 & & 3 & 1 \\
\hline & 177 & 16 & 4,5 & & & 1 & & & 30 & & 5 & 9 & 6 & & 2 & 2 & 15 & 2 & 7 & \\
\hline \multirow{3}{*}{$\begin{array}{l}\text { alluvions } \\
\text { des vallées } \\
\text { fossiles }\end{array}$} & 161 & 2 & 54 & & & & 1,5 & & 3 & & 24 & 9 & 1 & 1,5 & 1 & & & & 4 & \\
\hline & 163 & 34 & 32 & 2 & & & & 6 & & 6 & 8 & 2 & 2 & & & & & 4 & 2 & \\
\hline & 187 & 15 & 28 & 1 & 3 & 1 & & 3 & 3 & 6 & 6 & 14 & 5 & & 2 & 2 & 7,5 & & & 2 \\
\hline \multirow{6}{*}{$\begin{array}{l}\text { alluvions } \\
\text { Bouzanne }\end{array}$} & 181 & & 13 & & & & & & 40 & 0,7 & & 5,5 & 2 & 1,5 & 7 & 6 & 22 & & 2 & \\
\hline & 183 & & 16 & & & & & & 31 & & 4,5 & 10 & 4 & & 6,5 & 6,5 & 18 & & 4 & \\
\hline & 184 & 19 & 24 & 0,5 & 0,5 & & 0,7 & 0,7 & 21 & 1,5 & 8 & 1,5 & 1,5 & & 1,5 & 3,5 & 8 & & 5 & 1,5 \\
\hline & 185 & 4,5 & 25 & & & & 0,7 & 1 & 29 & & 8 & 7 & 7 & 0,7 & 3,5 & 6 & 2 & & 3 & \\
\hline & 186 & 1,5 & 57 & & & & & & 8 & & 6 & 12 & 1,5 & & 6 & 6 & 1,5 & & & \\
\hline & 187 & 15 & 28 & 1 & 3 & 1 & & 3 & 3 & 6 & 6 & 14 & 5 & & 2 & 2 & 7,5 & & & 2 \\
\hline \multirow{4}{*}{$\begin{array}{c}\text { alluvions } \\
\text { actuelles } \\
\text { Indre }\end{array}$} & 51 & 3 & 9 & & 1 & & 1 & & 67 & 2 & 13 & 1 & 0,5 & & & 5 & 7 & & & \\
\hline & 160 & 1 & 7,5 & & & & & & 60 & & 11 & 8 & & & 1 & 1 & 5 & & 5 & 1 \\
\hline & 166 & 14 & 26 & 1 & 1 & & 2 & 2 & 30 & & 6 & 3,5 & 6 & & 2 & 2 & 4 & & 2 & \\
\hline & 169 & 7,5 & 39 & 1,5 & & & & & 26 & & 1,5 & 6 & 3 & & 1,5 & & 12 & & 1,5 & \\
\hline \multirow{2}{*}{$\begin{array}{c}\text { alluvions } \\
\text { anciennes } \\
\text { Indre } \\
\end{array}$} & 168 & & 30 & 0,5 & 0,5 & & 0,5 & & 24 & 0,5 & 16,5 & 13 & 2 & & 2 & 6 & 1 & & 2 & 0,7 \\
\hline & 188 & 10 & 13 & & & & & 2,5 & 38 & 1 & 16 & 8,5 & 1 & & 5 & 1 & 1 & 1 & 1 & \\
\hline Claise & 196 & 35 & 35 & 1,7 & & & & 1,7 & & 7 & 3,5 & 7 & 9,6 & & & 1 & 3,5 & & & 3,5 \\
\hline
\end{tabular}

Tableau 3 : Composition en minéraux lourds des échantillons localisés sur les figures 2 et 3 et sur le tableau 2 Heavy minerals composition on samples located on figures 2 and 3 and on table 2

$25 \mathrm{~m}$ au-dessus du cours actuel. La ligne de partage des eaux entre l'Indre et la Bouzanne suit un tracé sinueux et atteint $178 \mathrm{~m}$ dans les trois vallées fossiles qui se raccordent à la moyenne terrasse de l'Indre (fig. 3). Aucun témoin alluvial n'a été trouvé en place dans ces vallées fossiles, toutefois, le grand nombre d'espèces minérales observé dans l'échantillon 187 suggère un passage fluvial. À une distance égale des sources, la Bouzanne est située à une altitude toujours plus basse 


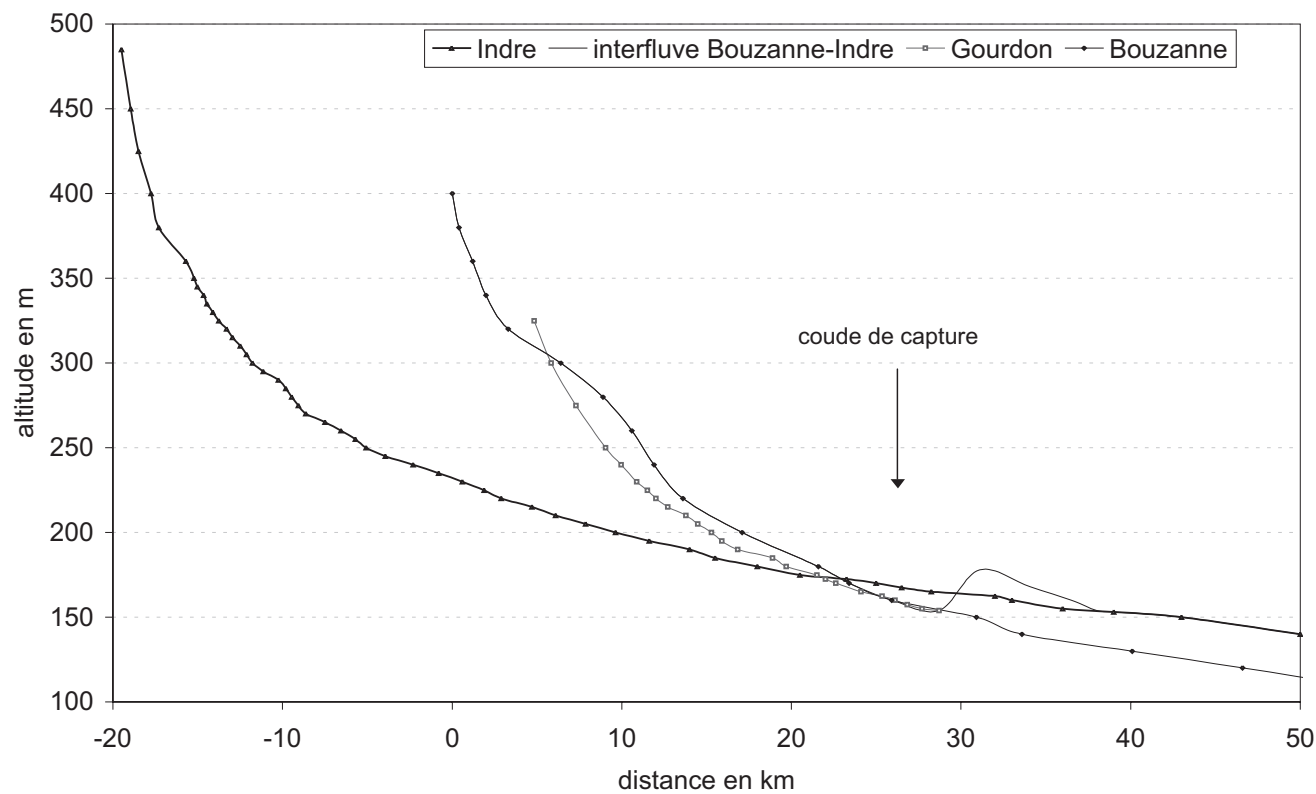

Figure 6 : Profils longitudinaux de l'Indre, de la Bouzanne, du Gourdon et de l'interfluve Bouzanne-Indre dans la zone de capture fluve in the capture zone

Longitudinal profiles of the Indre, Bouzanne and Gourdon rivers and of the Bouzanne-Indre inter-

que l'Indre et le différentiel augmente vers l'aval : 50 m à 29 km (confluence Bouzanne-Gourdon et La Châtre), $60 \mathrm{~m}$ à $60 \mathrm{~km}$.

Les indices de concavité diminuent d'est en ouest, de l'Indre $(0,72)$ au Creusançais $(0,35)$, en passant par le Gourdon $(0,59)$ et la Bouzanne amont $(0,48)$. Le graphique adimentionnel souligne bien ces différences de concavité et révèle les principales anomalies de pente (fig. 4). De l'amont à l'aval, la Bouzanne présente 4 principales ruptures de pente (figs. 2 et 7 ) : la première se développe dans le socle sur 1,3 km, entre 260 et $240 \mathrm{~m}$, la pente atteint 15,4\%o (indice SL = 163); la seconde se situe $6,5 \mathrm{~km}$ en amont de la confluence avec le Gourdon, dans le Toarcien marneux, elle mesure $1,8 \mathrm{~km}$ avec une pente de 5,6\%o et un indice SL de 120; la troisième, longue de $2,5 \mathrm{~km}$, correspond aux méandres localisés entre 150 et $140 \mathrm{~m}$ dans le calcaire bajocien (3,7\%o de pente et indice $\mathrm{SL}=114)$; la dernière se trouve dans les calcaires récifaux bajociens juste avant la confluence de la Creuse à $90 \mathrm{~m}$ (sur $2 \mathrm{~km}$, la pente atteint 5,8\%o et l'indice SL, 343). Sur le Gourdon, les ruptures de pente sont moins longues et moins prononcées : les indices SL se tiennent entre 72 et 90 (fig. 2). Deux ruptures de pente accidentent l'Indre de part et d'autre de la cuesta bajocienne (indices SL $=112$ et 128).

\section{Interprétations}

\section{Les Paléotracés du Pliocène supérieur}

L'unité de Fougères constituant la principale source d'andalousite, il convient d'admettre que les formations de la région d'Ardentes ont été alimentées par les flux venant de l'axe de la Creuse. En effet, les dépôts situés près de La Châtre (échantillon 19), qui jalonnent un paléotracé du Pliocène supérieur, ne renferment que très peu d'andalousite. Le cône d'épandage d'Ardentes, qui renferme des cryoturbations fossilisées par la partie supérieure de la formation (Freytet et al., 


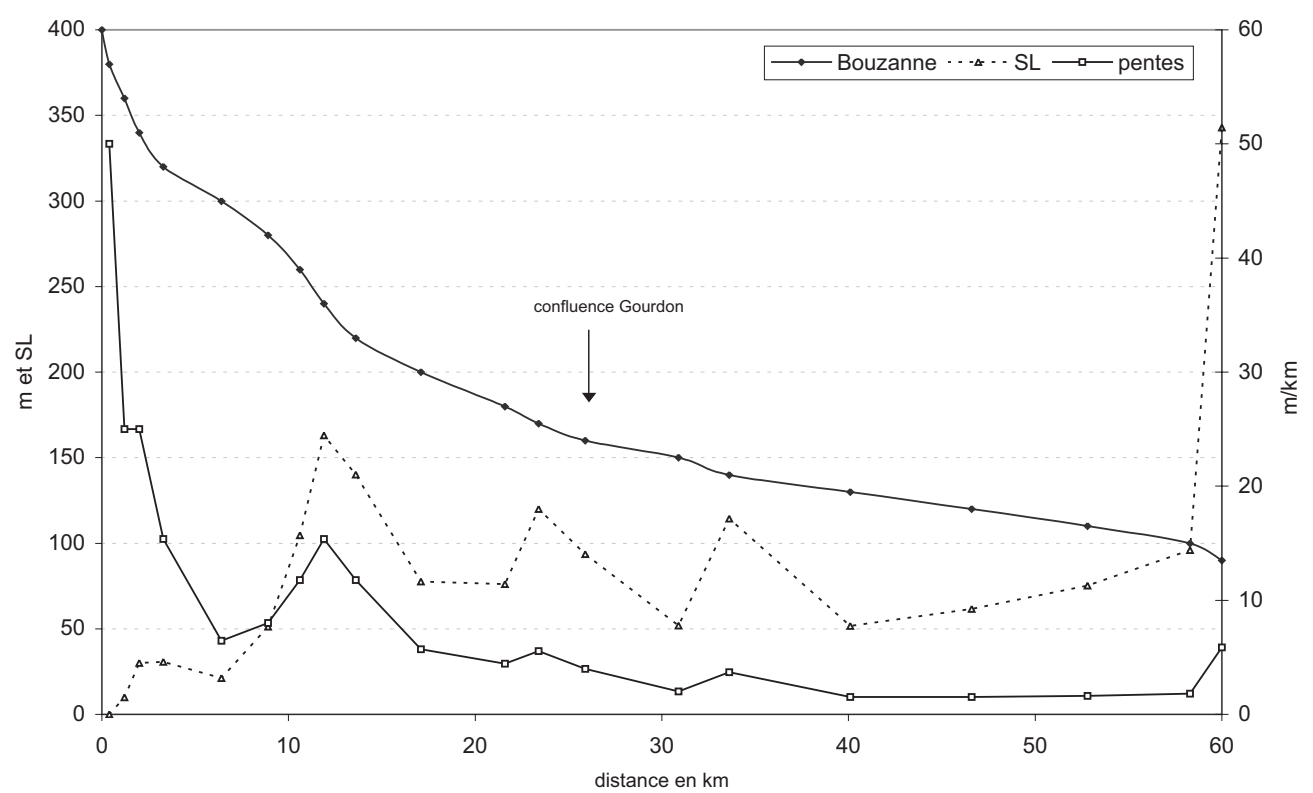

Figure 7 : Les ruptures de pente du profil de la Bouzanne. Pentes et indices SL (d'après Keller et Pinter, 1996) The Bouzanne knickzones. Gradients and SL index (after Keller and Pinter, 1996)

1986), a été placé dans le Pléistocène inférieur par J.-C. Yvard (1978), ce qui est en accord avec les datations R.P.E. (1,7 Ma) obtenues pour le lambeau du Pin (Despriée et al., 2004). Cependant ces formations ressemblent beaucoup à celles trouvées plus à l'ouest dans les gouttières de l'Arnon et du Cher et datées du Pliocène supérieur (3,4 à $2 \mathrm{Ma}$ ) par la présence de minéraux montdoriens (Larue et Étienne, 1998). En outre, le Pliocène supérieur a aussi enregistré des épisodes froids (Prétiglien) pouvant expliquer la présence de figures périglaciaires dans les dépôts. Il est possible que la forte altération de ces formations ait pu rajeunir les datations R.P.E., comme J. Despriée et al. (2004) l'ont d'ailleurs montré pour la nappe de 90 à $110 \mathrm{~m}$ d'altitude relative. Cet écoulement peu chenalisé a été interrompu par une phase de soulèvement de l'« accident sud du Bassin de Paris » qui a contraint la Creuse à traverser la Brenne selon le paléo-tracé 2 qui atteint la région de Buzançais. Les minéraux lourds des alluvions résiduelles conservées sur ce tracé (échantillon 194) indiquent des apports de la Creuse (andalousite, staurotide, disthène). Cet écoulement a été de courte durée, car la poursuite du soulèvement est à l'origine de la bifurcation vers l'ouest des principaux cours d'eau traversant la cuesta et cela dès le début de l'incision pléistocène.

\section{LES CAUSES ET LES IMPACTS GÉOMORPHOLOGIQUES DE LA CAPTURE PLÉISTOCÈNE DE LA BOUZANNE}

Les vallées fossiles (windgaps) témoignant de la capture de la Bouzanne se raccordent à la moyenne terrasse de l'Indre, ce qui suggère un soulèvement de l'« accident sud du Bassin de Paris » d'une quinzaine de mètres depuis l'abandon de la vallée. Cette surrection a conduit à des défluviations rendues possibles par le faible encaissement des vallées et par la faible résistance du substrat. Ainsi, le Gourdon, qui rejoignait l'Indre, a été dévié vers le cours S-N de la Bouzanne (fig. 2 et 3). Ensuite, l'écoulement s'est déversé vers la Bouzanne inférieure qui s'était rapprochée de la zone de défluviation grâce à la progression de l'incision régressive induite par le bas niveau de base constitué par la Creuse. Malgré des puissances fluviales supposées semblables, l'Indre et la Bouzanne ont réagi différemment au soulèvement de l'«accident sud du Bassin de Paris ». 
L'Indre, en réduisant la largeur de son lit dans le calcaire, a conservé une puissance spécifique suffisante pour que l'incision compense le soulèvement. En effet, la réduction de la largeur du lit permet aux cours d'eau de se maintenir dans une zone en surrection (Whittaker et al., 2007). En revanche, le creusement de la Bouzanne a été ralenti par l'abondance de la charge fournie par les dépôts détritiques tertiaires du bassin de Lys-Saint-Georges qu'elle devait traverser : les formes de défluviation conservées près de la ligne de partage des eaux actuelle (fig. 3) témoignent de lits larges à chenaux tressés qui traduisent une diminution de la puissance spécifique de l'écoulement.

On peut supposer que l'érosion régressive induite par la Creuse s'est propagée vers l'amont sur la Bouzanne et le Gourdon. Bien que la précision des cartes topographiques au 1/25000 ne soit que de $5 \mathrm{~m}$, le relevé de la position des knickpoints indique, qu'en $200 \mathrm{ka}$, la progression vers l'amont a été de $6 \mathrm{~km}$ environ, soit une vitesse de $3 \mathrm{~cm} / \mathrm{an}$, dans des roches tendres : argiles sableuses éocènes et marnes et argiles toarciennes (Lorenz et al., 1985; Lorenz et al., 1990). En revanche, dans les roches résistantes du Bajocien, le recul des ruptures de pente apparaît très lent et non mesurable (troisième et quatrième ruptures sur la Bouzanne). Comme l'ont montré P. Bishop et al. (2005), la vitesse de recul des knickpoints est aussi contrôlée par la puissance fluviale qui augmente avec la taille des bassins versants amont. En Espagne du sud, A. F. García et al. (2004) indiquent des vitesses semblables de 2,6 à 3,4 cm/an entre 303 ka et 3,5 ka sur le Rio Andarax. L'incision initiée par le niveau de base formé par la Creuse explique aussi les différences de concavité d'est en ouest : plus proche de la Creuse, le Creusançais a une faible concavité car il doit plus creuser que les cours d'eau plus éloignés pour atteindre un profil régularisé.

La vallée conquérante a subi une morphogenèse active depuis la capture, parce que le bassin amont s'est brutalement agrandi et que la pente forte située au point de capture a stimulé l'incision régressive. Au NW de Jeu-les-Bois, la Bouzanne a développé deux beaux méandres encaissés, de 700 m de longueur d'onde et d'amplitude, dans les dépôts éocènes mais aussi dans le substrat bajocien résistant formé de calcaire à entroques et silex. Ce secteur correspond à une knickzone $(\mathrm{SL}=$ 114) et les alluvions Fx (Weischsélien) peu épaisses forment une terrasse étagée par rapport à la plaine alluviale. À l'aval, dans le bassin d'Arthon-Velles, l'accumulation d'alluvions sablo-caillouteuses donne une vaste terrasse Fx qui domine la plaine alluviale d'une dizaine de mètres, mais les nappes sont ici emboîtées. Près de la confluence avec la Creuse, la pente augmente à nouveau $(\mathrm{SL}=342)$, trois méandres encaissés de $1000 \mathrm{~m}$ de longueur d'onde et de $900 \mathrm{~m}$ d'amplitude sont sculptés dans les calcaires récifaux sous-jacents aux dépôts sableux éocènes et les alluvions anciennes ne sont pas conservées.

Le bassin supérieur de l'Indre a été décapité par la capture, il a perdu presque la moitié de sa superficie (bassin supérieur de la Bouzanne actuelle), ce qui a eu des conséquences sur la dynamique fluviale du secteur aval. Avec un débit réduit de moitié, l'Indre a perdu une partie de sa puissance érosive, si bien que l'incision suivant le remblaiement de la nappe $F_{W}$ n'a pu atteindre le substrat. Il en résulte que les alluvions weichséliennes de l'accumulation suivante sont emboîtées dans celles de la nappe Fw saalienne (Debrand-Passard, 1972; Rasplus et al., 1989a), alors qu'elles sont étagées dans les autres vallées, la Creuse par exemple (Cohen-Julien et al., 1998). Les alluvions weichséliennes essentiellement sableuses sont aussi moins grossières que les précédentes qui sont plus caillouteuses. En analysant une situation semblable dans le Sud-Est espagnol, E. Maher et al. (2007) ont montré que la capture du Rio Alias s'était accompagnée d'une réduction de la taille des sédiments et d'une modification du style morphodynamique du cours d'eau décapité.

\section{Conclusion}

En couplant les analyses géomorphologique et sédimentologique (en particulier l'étude des minéraux lourds), il est possible de reconstituer les principaux paléoflux au cours du temps. Au Pliocène supérieur et au Pléistocène, des captures fluviatiles se sont produites dans le sud du Bassin parisien à la suite du rejeu d'accidents tectoniques tels que le dôme de Brion et l'« accident sud du Bassin de Paris », mais aussi en fonction de l'évolution du creusement des systèmes fluviaux. 
Plus faiblement encaissée que la Creuse, l'Indre a ainsi été décapitée par la capture des réseaux de la Petite Creuse, puis de la Bouzanne. Cette dernière capture, datée entre 200 et 280 ka à partir des terrasses de la Creuse, est donc plus récente que le pensaient P. Freytet et al. (1986). Elle montre que les impacts géomorphologiques sont importants aussi bien dans la vallée conquérante que dans le système décapité. Le recul des knickpoints développés suite à la capture s'est fait à des vitesses très variables en fonction de la résistance du substrat : $3 \mathrm{~cm}$ par an dans le Lias tendre et recul insignifiant dans les calcaires résistants du Bajocien. Ces ruptures de pente sont des anomalies très intéressantes à étudier car elles conservent bien les discontinuités du creusement provoquées par la tectonique et les fluctuations des niveaux de base relatifs. En outre, le maintien des cours d'eau dans une zone en soulèvement ne dépend pas seulement de la puissance fluviale, mais aussi de la lithologie qui contrôle la charge solide et la morphologie du lit.

\section{Bibliographie}

Bishop (P.), 1995. - « Drainage rearrangement by river capture, beheading and diversion », Progress in Physical Geography, vol. 19, p. 449-473.

Bishop (P.), Hoey (T. B.), Jansen (J. D.), ArtZA (I. L.), 2005. - « Knickpoint recession rate and catchment area: the case of uplifted rivers in eastern Scotland », Earth Surface Processes and Landforms, vol. 30, p. 767-778.

ChÂTEAuneuf (J.-J.), 1977. - «Datation palynologique du Tertiaire continental des bordures nord et ouest du Massif central », Bulletin du BRGM, vol. 1, n 4, p. 353-355.

Cohen-Julien (M.), Quénardel (J.-M.), Lerouge (G.), Lorenz (J.), Macaire (J.-J.), Freytet (P.), Maget (P.), Debrand-Passard (S.), 1998. - Feuille géologique (593) à 1/50 000 d'Argenton-sur-Creuse, Notice explicative, BRGM, Orléans, 167 p.

Davis (W. M.), 1895. - « La Seine, la Meuse et la Moselle », Annales de Géographie, vol.19, p. 25-49.

Debrand-PAssard (S.), 1972. - Feuille géologique (544) à 1/50 000 de Châteauroux. Notice explicative, BRGM, Orléans, 18 p.

Demoulin (A.), 1998. - « Testing the tectonic significance of some parameters of longitudinal river profiles : the case of the Ardenne (Belgium, NW Europe) », Geomorphology, vol. 24, p. 189-208.

Despriée (J.), Gageonnet (R.), Voinchet (P.), Bahain (J.-J.), Falguères (C.), Duvialard (J.), Varache (F.), 2004. - « Pleistocene fluvial systems of the Creuse River (Middle Loire Basin, Centre Region, France) », Quaternaire, $\mathrm{n}^{\circ} 1-2$, p. 77-86.

Freytet (P.), 1988. - «Quelques réflexions sur le relief du Limousin », Norois, n 138, p. 201-221.

Freytet (P.), Lerouge (G.), Lorenz (C.), Lorenz (J.), 1986. - «Intérêt de l'étude pluridisciplinaire d'une région; stratigraphie, géologie structurale, géomorphologie, néotectonique, télédétection du sud du bassin de Paris », Bull. Inf. Géol. Bass. Paris, vol. 23, n² 2, p. 3-15.

Freytet (P.), Lorenz (C.), Lorenz (J.), Macaire (J.-J.), 1989. - Connaissances nouvelles sur les déformations récentes (néotectonique) du sud du Bassin parisien et de sa bordure : exemple du sud-ouest du Berry, $114^{\mathrm{e}}$ Congrès national des Sociétés savantes, Géologie du bassin parisien, Éditions du Comité des travaux historiques et scientifiques, Paris, p. 181-201.

Freytet (P.), Lerouge (G.), Quénardel (J.-M.), Bogdanoff (S.), Bouvier (P.), Cohen-Julien (M.), Lemaire (D.), Rolin (P.), Schmitt (P.), 1985. - «Esquisse néotectonique des pays limousins, marchois et bourbonnais ( $\mathrm{N}$ du Massif central français) », Comptes Rendus de l'Académie des Sciences, Paris, série b II, p. 1163-1168.

García (A. F.), Zhu (Z.), Ku (T. L.), Chadwick (O. A.), Chacon Montero (J.), 2004. - « An incision wave in the geologic record. Alpujarran Corridor, southern Spain (Almería) », Geomorphology, vol. 60, p. 37 72 .

Keller (E. A.), Pinter (N.), 1996. - Active tectonics. Earthquakes, uplift and landscape, Prentice Hall, Upper Saddle River, N.J., 338 p. 
Lablanche (G.), Marchand (D.), Lefavrais-Raymond (A.), Debrand-Passard (S.), Gros (Y.), Debéglia (N.), Maget (P.), Lallier (D.), 1994. - Feuille géologique (572) au 1/50 000 de Saint-Amand-Montrond, Notice explicative, BRGM, Orléans, 81 p.

Langbein (W. B.), 1964. - «Profiles of rivers of uniform discharge », United States Geological Survey Professional Paper, n 501 B, p. 119-122.

LARUE (J.-P.), 2000. - « Les réorganisations hydrographiques du bassin du Cher dans le sud du Bassin parisien (France) », Géomorphologie : relief, processus, environnement, n² 2, p. 105-124.

—, 2005. - «Tectonique, érosion et hydrographie sur la bordure nord-ouest du Massif central (France) », Géomorphologie : relief, processus, environnement, n 4, p. 275-296.

—, 2008. - « Tectonic influences on the Quaternary drainage evolution on the north-western margin of the French Central Massif: the Creuse valley example », Geomorphology, vol. 93, p. 398-420.

LARue (J.-P.), Étienne (R.), 1998. - « Les formations détritiques miocènes, pliocènes et quaternaires entre le Massif central et la Sologne (France). Nouveaux éléments d'interprétation », Géologie de la France, $\mathrm{n}^{\circ} 1$, p. 39-56.

Lerouge (G.), 1984. - Contribution à l'étude de la fracturation du NW du Massif central et du sud du bassin de Paris (France), Thèse de $3^{\mathrm{e}}$ cycle, Université d'Orléans, 2 vol, 394 p.

Lerouge (G.), Freytet (P.), Lorenz (C.), Lorenz (J.), 1986. - «Proposition d'une chronologie des évènements tectoniques, sédimentaires et morphologiques néogènes et quaternaires dans le sud du Bassin de Paris et le nord-ouest du Massif central français », Comptes Rendus de l'Académie des Sciences, Paris, série II, t. 303, p. 1749-1752.

Lorenz (C.), Lorenz (J.), 1983. - « Mise en évidence d'un «Accident sud du Bassin de Paris » affectant le Paléozoïque entre Ancenis et Montluçon, à partir de ses manifestations tectoniques et sédimentaires dans la couverture ", Comptes Rendus de l'Académie des Sciences, Paris, série II, t. 297, p. 73-76.

—, 1985. - « Structuration du S du bassin de Paris en blocs basculés de direction sud-armoricaine », Comptes Rendus de l'Académie des Sciences, Paris, série II, t. 301, p. 239-241.

Lorenz (J.), Lorenz (C.), Debrand-Passard (S.), 1985. - Feuille géologique (571) à 1/50 000 d'Ardentes. Notice explicative, BRGM, Orléans, 12 p.

Lorenz (C.), Debrand-Passard (S.), Lorenz (J.), Desprez (N.), Manivit (J.), 1990. - Feuille géologique (570) à 1/50000 de Velles. Notice explicative, BRGM, Orléans, 35 p.

Mather (A. E.), 2000a. - «Adjustment of a drainage network to capture induced base-level change: an example from the Sorbas Basin, SE Spain », Geomorphology, vol. 34, 271-289.

—, 2000b. - «Impact of headwater river capture on alluvial system development », Journal of the Geological Society (London), vol. 157, p. 957-966.

Maher (E.), Harvey (A. M.), Derek (F.), 2007. - « The impact of a major Quaternary river capture on the alluvial sediments of a beheaded river system, the Rio Alias SE Spain », Geomorphology, vol. 84, p. 344-356.

Parfenoff (A.), Pomerol (C.), Tourend (J.), 1970. - Les minéraux en grains, méthodes d'étude et détermination, Masson, 578 p.

Rasplus (L.), Alcaydé (G.), Lablanche (G.), Macaire (J.-J.), 1989a. - Feuille géologique (543) à 1/50 000 de Buzançais. Notice explicative, BRGM, Orléans, 39 p.

Rasplus (L.), Lorenz (J.), Lorenz (C.), Macaire (J.-J.), 1989b. - Feuille géologique (569) à 1/50 000 de Saint-Gaultier. Notice explicative, BRGM, Orléans, 39 p.

Weisrock (A.), 1997. - «Avant-propos. Paléoréseaux hydrographiques quaternaires : centenaire W.M. Davis. Cent ans après Davis: la question des captures et les paléoréseaux hydrographiques quaternaires d'après les exemples de l'Europe du Nord-Ouest », Géographie Physique et Quaternaire, vol. 51, n 3, p. 261-266.

Whittaker (A. C.), Cowie (P. A.), Attal (M.), Tucker (G. E.), Roberts (G. P.), 2007. - « Bedrock channel adjustment to tectonic forcing: Implications for predicting river incision rates », Geology, vol. 35, p. 103-106.

Yvard (J.-C.), 1978. - Géomorphologie du sud-ouest du Bassin de Paris, Thèse d'État, Paris 4, 4 vol., $1174 \mathrm{p}$. 


\section{Remerciements}

Nous remercions S. Desruelles (Université Paris 12), pour la mise au net des figures, ainsi que les deux relecteurs anonymes et $S$. Suanez qui ont contribué à l'amélioration du manuscrit.

Cet article a été reçu le 20 novembre 2006 et définitivement accepté le 21 mai 2007. 